\title{
Numerical study using finite element method for the thermal response of fiber specklegram sensors with changes in the length of the sensing zone
}

\author{
J.D. Arango ${ }^{1}$, Y.A. Vélez ${ }^{1}$, V.H. Aristizabal ${ }^{2}$, F.J. Vélez ${ }^{2}$, J.A. Gómez ${ }^{3}$, J.C. Quijano ${ }^{3}$, J. Herrera-Ramirez ${ }^{1}$ \\ ${ }^{1}$ Facultad de Ingenierías, Instituto Tecnológico Metropolitano, Medellín, Colombia, \\ ${ }^{2}$ Facultad de Ingeniería, Universidad Cooperativa de Colombia, Medellín, Colombia, \\ ${ }^{3}$ Grupo de investigación de Física Básica y Aplicada, Politécnico Colombiano Jaime Isaza Cadavid, Medellín, Colombia
}

\begin{abstract}
The response of fiber specklegram sensors (FSSs) is given as function of variations in the intensity distribution of the modal interference pattern or speckle pattern induced by external disturbances. In the present work, the behavior of a FSS sensing scheme under thermal perturbations is studied by means of computational simulations of the speckle patterns. These simulations are generated by applying the finite element method (FEM) to the modal interference in optical fibers as a function of the thermal disturbance and the length of the sensing zone. A correlation analysis is per-formed on the images generated in the simulations to evaluate the dependence between the changes in the speckle pattern grains and the intensity of the applied disturbance. The numerical simulation shows how the building characteristic of the length of sensing zone, combined with image processing, can be manipulated to control the metrological performance of the sensors.

Keywords: fiber optics sensors, computational electromagnetic methods, numerical approximation and analysis, optical sensing and sensors, speckle interferometry.

Citation: Arango JD, Vélez YA, Aristizabal VH, Vélez FJ, Gómez JA, Quijano JC, HerreraRamirez J. Numerical study using finite element method for the thermal response of fiber specklegram sensors with changes in the length of the sensing zone. Computer Optics 2021; 45(4): 534540. DOI: 10.18287/2412-6179-CO-852.

Acknowledgments: This work was partially funded by the Instituto Tecnológico Metropolitano (grant P20215), the Politécnico Jaime Isaza Cadavid (grant 2020/00132/001) and the Universidad Cooperativa de Colombia (grant INV2903). Y. A. Vélez also thanks the support given to her by the Instituto Tecnológico Metropolitano through its program of young researchers.
\end{abstract}

\section{Introduction}

The developments in FSS (Fiber Specklegram Sensor) that have been taking place in recent years, have positioned it as a valuable alternative for sensing multiple variables, given its characteristics such as small size, low comparative cost and its metrological properties that allow configuring different monitoring systems [1-9]. These types of sensors are conceived as devices that respond to the alterations induced on a multimode fiber by different external physicochemical variables (temperature, pressure, deformation, concentrations) [10-14] through changes in the intensity distribution in the modal interference pattern at the fiber output. At an experimental level, sensing schemes are developed using monomulti-mono mode fiber configurations, where the third component works as a filter that admits part of the light from the speckle pattern generated by the multimodal fiber, which allows configuring interrogated FSS schemes by variation of optical power. This technique is quite simple and inexpensive, although it misuses part of the spatial information of the speckle patterns associated with the state of the sensing fiber [15-19].

To evaluate the response of the FSS sensor, various investigations are supported by digital image analysis, whose algorithms allow the analysis of multiple records and estimate the variations induced in the speckle patterns in the face of external disturbances [2, 20-24]. Therefore, when alterations are presented in variables such as temperature, changes will be induced that can be estimated through correlation analysis carried out on the interference patterns from the sensing system. In this line, studies are reported that analyze the patterns captured by CCD cameras at the output of the multimode fiber, which are performed digital processing and correlation analysis in selected regions of said images [25, 26].

An alternative to make the analysis of these optical sensing systems more efficient consists in the generation of multiple speckle patterns, corresponding to modal interference patterns, through computational models of propagation in optical waveguides under external disturbances [27], in order to have a large digital image bank for study, and thus establish the characteristics of the sensor system in the face of induced changes in speckle patterns. In this sense, the finite element method (FEM) is configured as a powerful simulation tool in the generation of optical systems solutions, in particular, for propagation and interference processes in FSS under controlled disturbances [28, 29]. In consideration of the above, this work is oriented to study the response of a FSS, throw the analysis of the speckle patterns generated by FEM simulations, when the sensor is subjected to focused thermal 
disturbances. The evaluation of the sensor response is established by means of a correlation analysis discriminated in regions within these simulated images.

\section{Thermal disturbances representation}

To obtain the speckle pattern at the end of a multimode optical fiber (MOF) under a given temperature, the propagation of the electric field $\overrightarrow{\mathrm{E}}$ of each mode must be calculated by solving the vector wave equation:

$$
\nabla \times \nabla \times \overrightarrow{\mathrm{E}}(x, y, z)-k_{0}^{2} n^{2} \overrightarrow{\mathrm{E}}(x, y, z)=0,
$$

where $k_{0}=2 \pi / \lambda_{0}$ is the wave number in vacuum, $\lambda_{0}$ the wavelength in vacuum, and $n$ is the refractive index of the fiber. This refractive index is given by

$$
n=\left[\begin{array}{ccc}
n_{x} & 0 & 0 \\
0 & n_{y} & 0 \\
0 & 0 & n_{z}
\end{array}\right],
$$

where $n_{\mathrm{x}}, n_{\mathrm{y}}$ and $n_{\mathrm{z}}$ are found through the change of refractive indices $[10]$ :

$$
\Delta n_{i}=n_{i}-n_{0}=\Delta n_{i}^{T M}+\Delta n_{i}^{T O} \text {, with } i=x, y, z .
$$

In equation (3), $\Delta n_{i}^{T M}$ is the change in the refractive index due to thermo-mechanical disturbance (changes by material expansion due to temperature variations) and $\Delta n_{i}^{T O}$ is the change in refractive index due to thermooptical disturbance (changes due to variations in temperature). Then, using (3) we obtain:

$$
\begin{aligned}
& n_{x}=n_{0}+\left[C_{T O}-\frac{n_{0}^{3}}{2} \alpha\left(P_{11}+P_{12}\right)\right]\left(T-T_{0}\right), \\
& n_{y}=n_{0}+\left[C_{T O}-\frac{n_{0}^{3}}{2} \alpha\left(P_{11}+P_{12}\right)\right]\left(T-T_{0}\right), \\
& n_{z}=n_{0}+\left[C_{T O}-\frac{n_{0}^{3}}{2} 2 \alpha P_{12}\right]\left(T-T_{0}\right),
\end{aligned}
$$

where $C_{T O}$ stands for the thermo-optic coefficient, $\alpha$ for the coefficient of linear thermal expansion, $P_{11}$ and $P_{12}$ for the strain-optic coefficients of the material, $T_{0}$ for the temperature of the material at the initial moment (room temperature), and $n_{0}$ for the refractive index at the initial moment where the material is isotropic. For fused silica at $\lambda_{0}=632.8 \mathrm{~nm}$, the $C_{T O}=11.9 \times 10^{-6} /{ }^{\circ} \mathrm{C}[29,30]$, $\alpha=0.5 \times 10^{-6} /{ }^{\circ} \mathrm{C}, P_{11}=0.113$, and $P_{12}=0.252$ [31]. In the core, $n_{0}=n_{0 c o}=1.4570$, calculated using the Sellmeier's equation [28]:

$$
\begin{aligned}
& n_{0 c o}^{2}(\lambda[\mu m])=1+\frac{0.6961663 \lambda_{0}^{2}}{\lambda_{0}^{2}-0.0684043^{2}}+ \\
& +\frac{0.4079426 \lambda_{0}{ }^{2}}{\lambda_{0}{ }^{2}-0.1162414^{2}}+\frac{0.8974794 \lambda_{0}{ }^{2}}{\lambda_{0}{ }^{2}-9.896161^{2}} .
\end{aligned}
$$

For the cladding of the fiber $n_{0}=n_{0 \text { cla }}=1.4482$. This value is calculated using equation (6) with a numerical aperture $N A=0.16$ for the simulated fiber:

$$
n_{0 c l a}=\sqrt{n_{0 c o}^{2}-N A^{2}} .
$$

Note that the terms in equations (4), where the strainoptic coefficients are contained, can be neglected because these are much smaller than $C_{T O}$ in the case of fused silica, obtaining the following simplification:

$$
n_{x}=n_{y}=n_{z} \approx n_{0}+C_{T O}\left(T-T_{0}\right) .
$$

Equation (7) shows that the index of fused silica exhibits approximately an isotropic behavior with changes in temperature.

\section{Finite Element Method for numerical calculations of propagation modes}

If we assume an optical fiber of infinite length with a refractive index invariant along the propagation axis, a 2.5D approximation of the problem is obtained. This approach consists of finding the state of the system in a plane and then propagating it in the normal direction. In this case, the field can be expressed as $\overrightarrow{\mathrm{E}}(x, y, z)=e^{i \beta z} \overrightarrow{\mathrm{E}}(x, y)$, where $\beta$ is the propagation constant of the mode associated with $\overrightarrow{\mathrm{E}}(x, y, z)$.

Here, the electric field $\overrightarrow{\mathrm{E}}(x, y, z)$ of each mode can be calculated by solving equation (1) numerically through the finite element method (FEM) (see more details in [27, $28,33])$. To calculate the field $\overrightarrow{\mathrm{E}}(x, y)$ by FEM, the cross section of the fiber in a reference plane, that is taken as $z=0$, is discretized into triangular elements. Equation (1) is solved in each of these elements and then they are assembled to obtain the general solution for each mode (see fig. 1a).

(a)

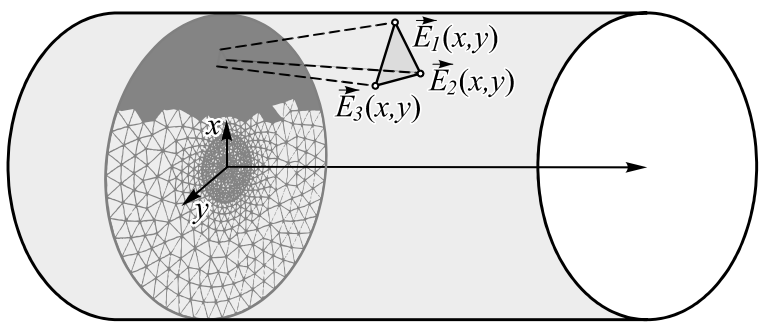

(b)
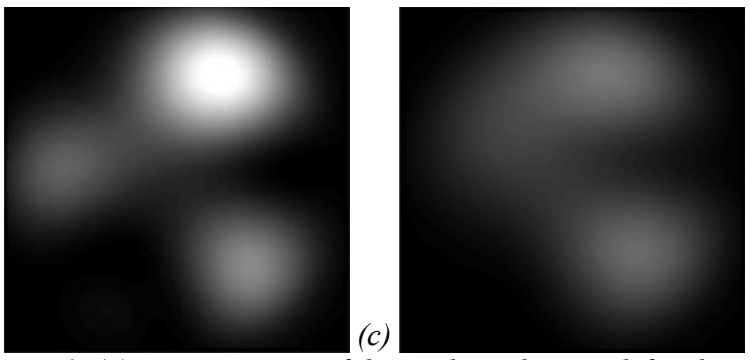

Fig. 1. (a) Discretization of the analysis domain defined for a MOF using a mesh of triangular elements for the implementation of the FEM. (b) and (c) are the numerically simulated specklegrams, $I_{\text {ref }}$ and $I_{n}$, obtained

for the temperatures of $30^{\circ} \mathrm{C}$ and $90^{\circ} \mathrm{C}$, respectively

In this way, we obtain the vector field $\overrightarrow{\mathrm{E}}(x, y)$ and the propagation constants of each mode in the plane $z=0$. Afterward, each mode is propagated along a given $z$, by computing the expression $e^{i \beta z} \overrightarrow{\mathrm{E}}(x, y)$. The vectorial su- 
perposition of all these modes produces the speckle pattern in the required $z$-plane [28].

\section{Analysis of specklegrams}

Since the changes in intensity due to thermal disturbances are visible at a local scale approximately close to that of the grain size of the speckle pattern, these changes cannot be properly quantified by averaging the intensity of the entire pattern. Therefore, when there are speckle patterns at the output of a MOF, correlation techniques are usually employed to quantify the changes due to these disturbances. This technique is carried out through the algebraic implementation of the correlation coefficient given by [21]:

$$
C=\frac{\sum_{i} \sum_{j}\left(\left(I_{\text {ref }}(i, j)-\left\langle I_{\text {ref }}\right\rangle\right) \cdot\left(I_{n}(i, j)-\left\langle I_{n}\right\rangle\right)\right)}{\sqrt{\left(\sum_{i} \sum_{j}\left(I_{\text {ref }}(i, j)-\left\langle I_{\text {ref }}\right\rangle\right)^{2}\right)\left(\sum_{i} \sum_{j}\left(I_{n}(i, j)-\left\langle I_{n}\right\rangle\right)^{2}\right)}},
$$

where $\left\langle I_{r e f}\right\rangle$ is the average intensity of the speckle pattern at the fiber output in a reference state (fig. $1 b$ ) and $\left\langle I_{n}\right\rangle$ is the equivalent in a disturbed state (fig. 1c).

\section{Simulated system}

The simulated system is an FSS with a mono-multi mode fibers arrangement, with the multimode fiber acting as transducer. The simulated multimode fiber of fused silica is subjected to thermal disturbances and variation in some of its metrological parameters.

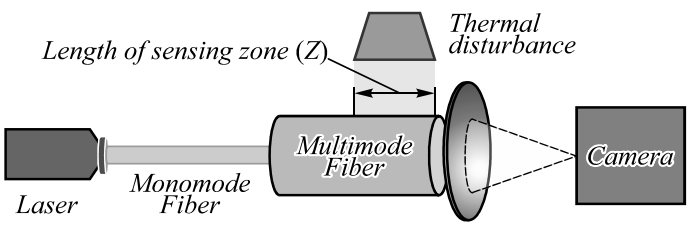

Fig. 2. Scheme of the simulated system

Fig. 2 shows the simulated system using a $632.8 \mathrm{~nm}$ laser, a MOF with core diameter of $9 \mu \mathrm{m}$, and 0.16 of $N A$. We established a room temperature of $24^{\circ} \mathrm{C}$. All these values are fixed while we carried out the simulations on changing disturbance temperature and length of the sensing zone.

The thermal effects and the light propagation for the generation of the speckle patterns in the MOF detailed above are simulated following the procedure described in sections 1 and 2, respectively. Here, a script in Matlab that integrates Comsol Multiphysics is implemented for the numerical solution of Eq. (1) by means of FEM (Finite Element Method), where first, the refractive index changes due to thermic disturbance are calculated through (7). Second, this computed refractive index is replaced in the wave equation (1) which is solved by FEM for the fields $\overrightarrow{\mathrm{E}}(x, y, z)$ of the propagating modes support by the MOF in the plane $z=0$, that is where the zone of thermal disturbance begins (see figs. 1a,2). Third, these computed fields are propagated a distance $z=Z$. Fourth, all these computed modes are vectorially added at the end of the MOF, producing the speckle pattern, as detailed in section 2 . Finally, this specklegram at the fiber output are analyzed using the correlation coefficient defined in equation (8).

\section{Specklegram analysis and sensor response}

Fig. 3 illustrates the effects that the variation of $N A$ has on the resulting specklegrams. It shows that by increasing the value of $N A$ the number of speckle grains in- creases significantly, as more modes can enter and propagate in the fiber. This is an example of the potential of these simulations to study the behavior of the sensor by modifying certain parameters. However, in this work we are centeaz the influence of the length of the sensing zone on the metrological parameters of the FSS. For this analysis we have selected an $N A$ of 0.16 .

Fig. 4 shows the correlation curves when changing the thermal disturbance, for different lengths of the sensing zone (Z). Fig. 4a. compares the correlation curves and shows how the correlation coefficient undergoes through a somehow periodic behavior as temperature changes, for five different $\mathrm{Z}$ lengths. As the $\mathrm{Z}$ length increases, the pseudo period of these curves reduces, showing more sensitivity, i.e., faster changes in correlation. Additionally, these curves seem as having an attenuating envelope.

As a plausible interpretation of this behavior, we think that this evolution of the correlation curve is governed by how the changes of the index of refraction as temperature changes affect the different modes propagating in the fiber. Dividing the propagating modes in the fiber into two broad groups of modes, i.e., one group of modes where the refractive index change will only represent a phase change, and another group where index change will represent an exclusion or inclusion as a propagating mode. Thus, modes that are only affected in phase would account for the periodic appearance of the curve, as the intensity of their superposition would be changing periodically and so the correlation curve. On the other hand, the other modes would account for the apparent attenuation on the correlation curve, as their inclusion or exclusion would affect the intensity of the specklegram in an uncorrelated way with respect to the reference.

Also, accordingly to the former interpretation, for an increase in $\mathrm{Z}$, the phase variation of the propagating modes with the change of temperature would be faster because of the cumulative effect, thus reducing the apparent period of the corresponding correlation curve.

Fig. $4 b$. to $4 f$. show the correlation curves individually. Isolating them allows an easy visualization of the dynamic range and sensitivity for the linear parts of these curves. It can be inferred from these figures that by increasing the length of the sensor zone, not only does the sensitivity of the sensor increase, but as mentioned, it also means a fast change in the overall appearance of the 
specklegram reducing this zone of linear behavior. This $\mid$ resulting in a decreased dynamic range of the system.

(a)

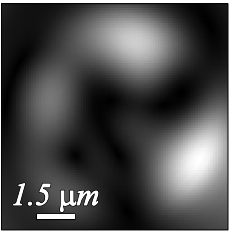

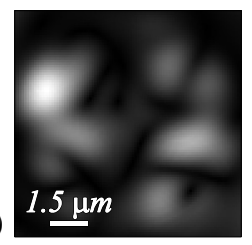

Fig 3. Influenc

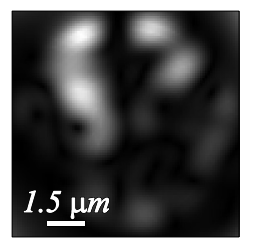

(d)
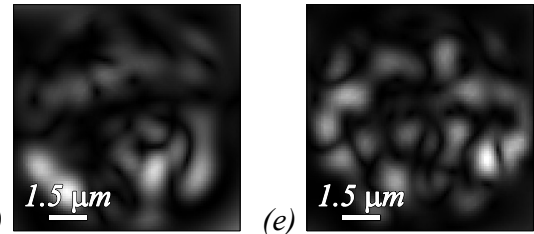

(a) $N A=0.10$, (b) $N A=0.15$, (c) $N A=0.20$, (d) $N A=0.25$, (e) $N A=0.30$

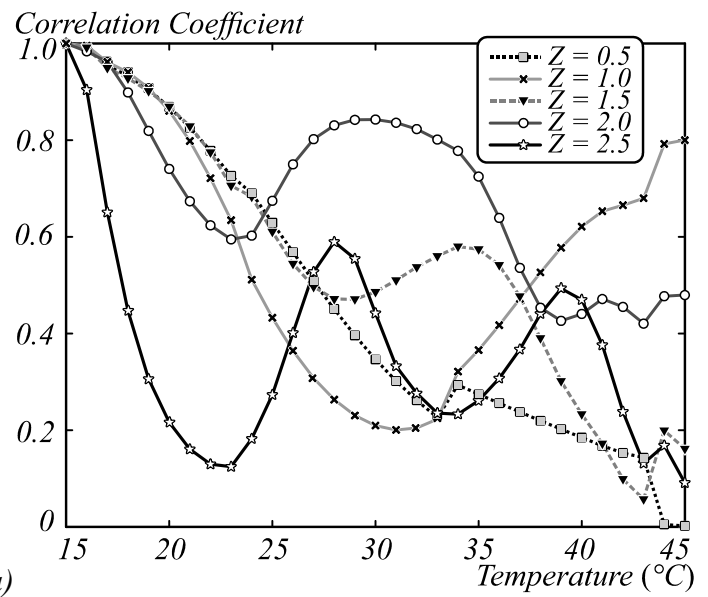

Correlation Coefficient

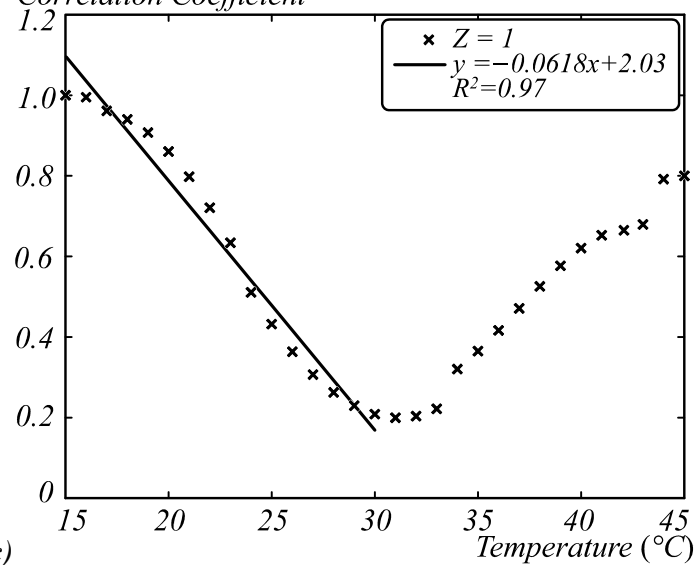

(c)

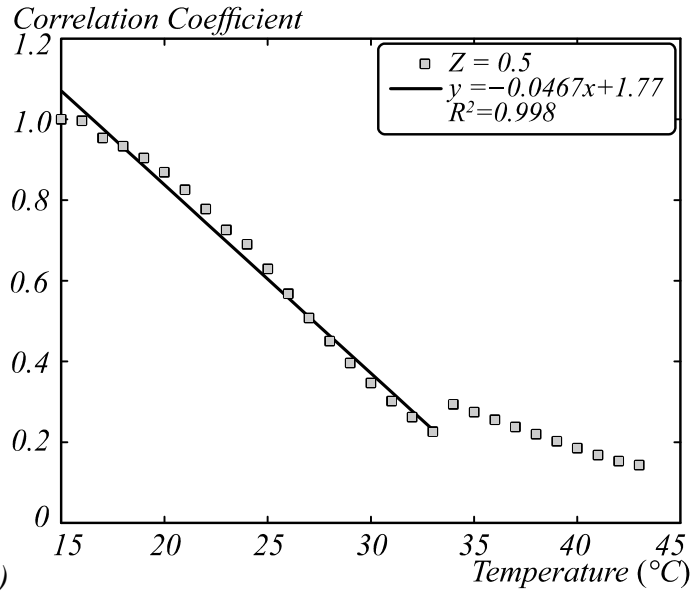

Correlation Coefficient

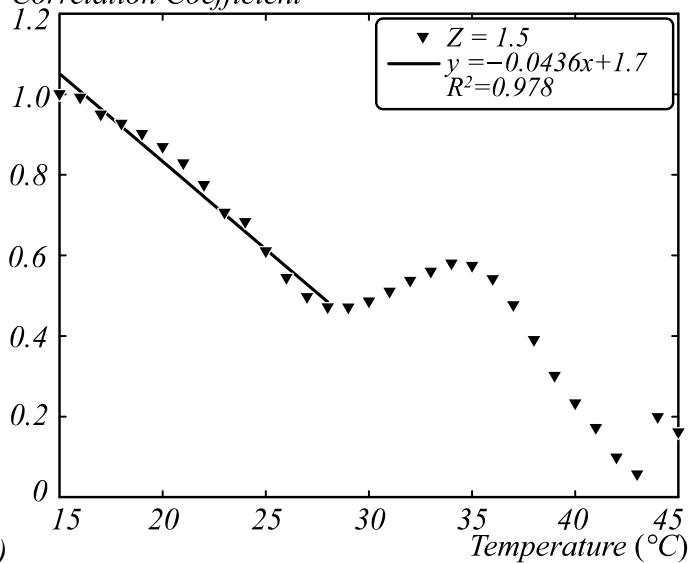

Correlation Coefficient

Correlation Coefficient
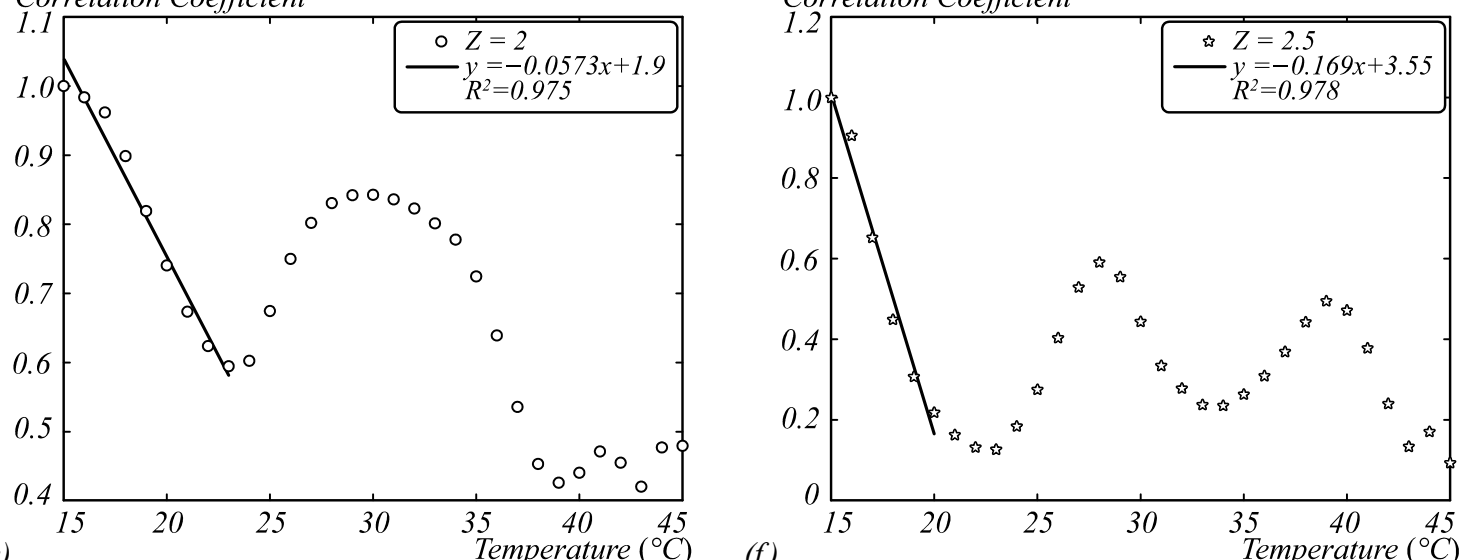

ig. 4. Correlation curves for different sensing zone lengths and linear fitting for the valid interval: (a) comparison for Zs from $0.5 \mathrm{~mm}$ to $2.5 \mathrm{~mm}$, (b) $Z=0.5 \mathrm{~mm}$, (c) $Z=1.0 \mathrm{~mm}$, (d) $Z=1.5 \mathrm{~mm}$, (e) $Z=2.0 \mathrm{~mm}$, and (f) $Z=2.5 \mathrm{~mm}$

Dynamic range, sensitivity, and linearity are metrological parameters of great importance in the definition of sensor performance. These simulations allow us to ascertain these parameters in an easy way. In Table 1 we show 
the data obtained in the numerical calculations for each length of sensing zone. We applied a linear fitting to the data points in the valid interval and extracted the dynamic range as $\Delta T=T_{\max }-T_{\min }$, the sensitivity $S$ as the slope of the linear fitting, and $R^{2}$ as a measure of the linearity. As mentioned, the data show more quantitatively that the dynamic range and sensitivity are strongly affected by the increase in Z, regularly increasing the sensitivity, but reducing the dynamic range. For $Z=1 \mathrm{~mm}$ there is a high sensitivity, even higher than for the two following $Z$ lengths. This shows that these metrological values are also dependent on the processing and selection of zone of correlation calculation. The $R^{2}$ values show a good linearity for the valid interval of data.

The correlation measure is dependent on how large the changes in the intensity distribution of the pattern are. Large changes in pattern intensity can result in behaviors such as pseudo periodic shapes shown before or discontinuity of correlation curves. One proposal to avoid these non-monotonic drawbacks is to redefine the areas where the calculation of the correlation curve is performed.

Table 1. Dynamic range, Sensitivity, and linearity by $Z$ variations

\begin{tabular}{cccc}
\hline $\begin{array}{c}\text { Length of sensing } \\
\text { zone } \mathbf{Z}(\mathbf{m m})\end{array}$ & $\begin{array}{c}\text { Dynamic range } \\
\boldsymbol{\Delta} \boldsymbol{T}\left({ }^{\circ} \mathbf{C}\right)\end{array}$ & $\begin{array}{c}\text { Sensitivity } \\
\boldsymbol{S}\left(\mathbf{1} /{ }^{\circ} \mathbf{C}\right)\end{array}$ & \multirow{2}{*}{$\mathbf{R}^{\mathbf{2}}$} \\
\hline \hline 0.5 & 18 & 0.04667 & 0.988 \\
1.0 & 15 & 0.06187 & 0.970 \\
1.5 & 13 & 0.04353 & 0.978 \\
2.0 & 8 & 0.05729 & 0.975 \\
2.5 & 5 & 0.16904 & 0.978 \\
\hline \hline
\end{tabular}

\section{Selection of region of interest (ROI)}

This strategy uses reduced areas and avoids the uncontrolled influence of all the pixels in the image, by selecting regions where the behavior of speckles is more monotonical. Since some of the ROIs improve the metrological parameters and others can yield the opposite result, the location and size of these ROIs cannot be predicted a priori and must be established by searching them through the speckle patterns.

Fig. 5 shows the result of applying this strategy to the numerical results for 3 different lengths of sensing zone (Z). It is visible from Fig. $5 a$ that there is an improvement in the dynamic range to the point that for the $3 \mathrm{Zs}$ analized, they overpass the larger range in the curves in the Figure 4. It is also evident the change in the sensitivity with the use of ROIs. Fig. $5 b$ illustrates the locations of the correspondent ROIs to each $\mathrm{Z}$ length, over the entire area of the speckle pattern. This shows that for an expected behavior, the ROI must be determined individually.

Table 2. shows the data of sensitivity and linearity for the curves in Fig. $5 a$. Although, the values in sensitivity are dependent in the selected ROI, the tendency of higher sensitivities for higher Zs still holds. Also, the use of these ROIs yields good linearities represented in $R^{2}$ values over 0.9 . As it is shown by the results for $Z=4 \mathrm{~mm}$, the selection of ROIs where low quantity of speckle grains is present can also help to control dynamic range, but with the associated trade-off of low sensitivity.

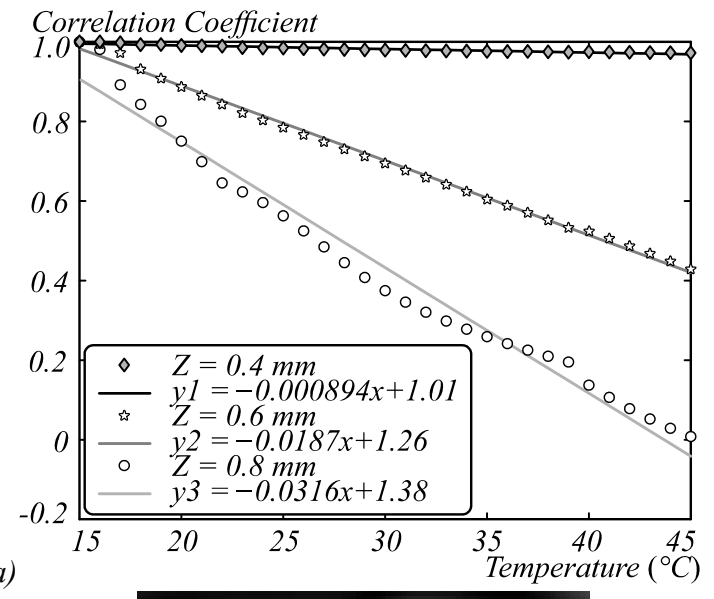

(a)

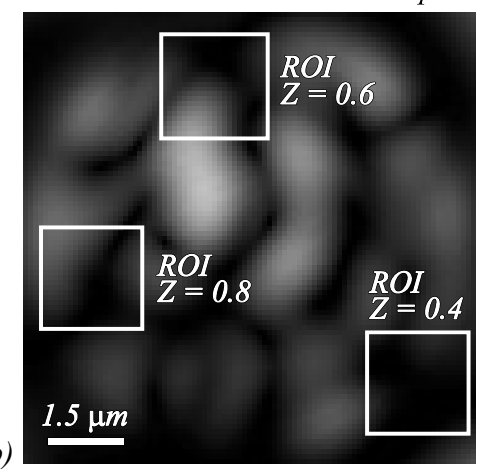

Fig. 5. (a) Correlation curves for different ROIs and sensing zone lengths. b) Location of the selected ROIs for the three $Z$

Table 2. Sensitivity and dynamic range by $Z$ variations

\begin{tabular}{ccc}
\hline $\begin{array}{c}\text { Length of sensing } \\
\text { zone } \mathbf{Z}(\mathbf{m m})\end{array}$ & $\begin{array}{c}\text { Sensitivity } \\
\boldsymbol{S}\left(\mathbf{1} /{ }^{\mathbf{C}}\right)\end{array}$ & $\boldsymbol{R}^{\mathbf{2}}$ \\
\hline \hline 0.4 & 0.000894 & 0.903 \\
0.6 & 0.018700 & 0.996 \\
0.8 & 0.031600 & 0.977 \\
\hline \hline
\end{tabular}

\section{Conclusions}

We showed that the FEM method for the numerical simulation of physical-optical phenomena in optical fibers can be a powerful tool in the study and design of FSSs. The numerical simulation of thermal disturbances applied to an FSS allowed us to show in a convenient way how the building characteristic of the length of sensing zone, combined with image processing, can be manipulated to control the metrological performance of the sensors. Over the correlation curves we showed improvements in dynamic range, linearity, and sensitivity. This work opens the possibility to consolidate a costeffective methodology for designing this kind of sensors.

\section{References}

[1] Campanella CE, Cuccovillo A, Campanella C, Yurt A, Passaro VMN. Fibre Bragg Grating based strain sensors: Review of technology and applications. Sensors 2018; 18(9): 3115. DOI: 10.3390/s18093115. 
[2] Gómez JA, Salazar Á. Self-correlation fiber specklegram sensor using volume characteristics of speckle patterns. Opt Lasers Eng 2012; 50(5): 812-815. DOI: 10.1016/j.optlaseng.2012.01.002.

[3] Goodman JW. Speckle phenomena in optics: Theory and applications. $2^{\text {nd }}$ ed. Bellingham: SPIE Press; 2020.

[4] Leal-Junior AG, Frizera A, Marques C, Pontes MJ. Optical fiber specklegram sensors for mechanical measurements: A review. IEEE Sens J 2020; 20(2): 569-576. DOI: 10.1109/JSEN.2019.2944906.

[5] Wu S, Yin S, Yu FTS. Sensing with fiber specklegrams. Appl Opt 1991; 30(31): 4468-4470. DOI: 10.1364/AO.30.004468.

[6] Efendioglu HS. A review of fiber-optic modal modulated sensors: Specklegram and modal power distribution sensing. IEEE Sens J 2017; 17(7): 2055-2064. DOI: 10.1109/JSEN.2017.2658683.

[7] Cabral TD, Fujiwara E, Warren-Smith SC, EbendorffHeidepriem H, Cordeiro CMB. Multimode exposed core fiber specklegram sensor. Opt Lett 2020; 45(12): 32123215. DOI: 10.1364/OL.391812.

[8] Qian S, Xu Y, Zhong L, Su L. Investigation on sensitivity enhancement for optical fiber speckle sensors. Opt Express 2016; 24(10): 10829-10840. DOI: 10.1364/OE.24.010829.

[9] Wu P, Zhu S, Hong M, Chen F, Liu H. Specklegram temperature sensor based on femtosecond laser inscribed depressed cladding waveguides in Nd:YAG crystal. Opt Laser Technol 2019; 113: 11-14. DOI: 10.1016/j.optlastec.2018.12.004.

[10] Castaño LF, Gutiérrez LC, Quijano JC, Herrera-Ramírez JA, Hoyos A, Vélez FJ, et al. Temperature measurement by means of fiber specklegram sensors (FSS). Opt Pura y Apl 2018; 51(3): 1-7. DOI: 10.7149/OPA.51.3.50306.

[11] Feng F, Chen W, Chen D, Lin W, Chen SC. In-situ ultrasensitive label-free DNA hybridization detection using optical fiber specklegram. Sensors Actuators, B Chem 2018; 272: 160-165. DOI: 10.1016/j.snb.2018.05.099.

[12] Gåsvik KJ. Optical metrology. Chichester: John Wiley and Sons Ltd; 2002. ISBN: 0-470-84300-4.

[13] Hoyos A, Gómez ND, Gómez JA. Fiber specklegram sensors (FSS) for measuring high frequency mechanical perturbations. Proc SPIE 2013; 8785: 8785BH. DOI: 10.1117/12.2026075.

[14] Fujiwara E, Da Silva LE, Cabral TD, De Freitas HE, Wu YT, Cordeiro CMDB. Optical fiber specklegram chemical sensor based on a concatenated multimode fiber structure. J Light Technol 2019; 37(19): 5041-5047. DOI: 10.1109/JLT.2019.2927332.

[15] Aristizabal VH, Hoyos A, Rueda E, Gomez ND, Gomez JA. Effect of wavelength on metrological characteristics of nonholographic fiber specklegram sensor. Photonic Sensors 2015; 5(1): 1-5. DOI: 10.1007/s13320-014-0210-3.

[16] Zhang Z, Ansari F. Fiber-optic laser speckle-intensity crack sensor for embedment in concrete. Sensors Actuators A Phys 2006; 126(1): 107-111. DOI: 10.1016/j.sna.2005.10.002.

[17] Darío Gómez N, Gómez JA. Effects of the speckle size on non-holographic fiber specklegram sensors. Opt Lasers Eng 2013; 51(11): 1291-1295. DOI: 10.1016/j.optlaseng.2013.05.007.
[18] Rodriguez-Cobo L, Lomer M, Lopez-Higuera JM. Fiber specklegram sensors sensitivities at high temperatures. Proc SPIE 2015; 9634: 96347J. DOI: 10.1117/12.2194288.

[19] Wang JJ, Yan SC, Ruan YP, Xu F, Lu YQ. Fiber-optic point-based sensor using specklegram measurement. Sensors 2017; 17(10): 2429. DOI: 10.3390/s17102429.

[20] Fujiwara E, Ri Y, Wu YT, Fujimoto H, Suzuki CK. Evaluation of image matching techniques for optical fiber specklegram sensor analysis. Appl Opt 2018; 57(33): 9845-9854. DOI: 10.1364/AO.57.009845.

[21] Gubarev F, Li L, Klenovskii M, Glotov A. Speckle pattern processing by digital image correlation. MATEC Web Conf 2016; 48: 04003. DOI: 10.1051/matecconf/20164804003.

[22] Liu Y, Li G, Qin Q, Tan Z, Wang M, Yan F. Bending recognition based on the analysis of fiber specklegrams using deep learning. Opt Laser Technol 2020; 131: 106424. DOI: 10.1016/j.optlastec.2020.106424.

[23] Gutiérrez LC, Castaño LF, Gómez JA, Quijano JC, Herrera-Ramírez JA, Hoyos A, et al. Specklegramas de fibra óptica analizados mediante procesamiento digital de imágenes. Rev La Acad Colomb Ciencias Exactas, Físicas y Nat 2018; 42(163): 182. DOI: 10.18257/raccefyn.608.

[24] Liu Y, Qin Q, Liu H-h, Tan Z-w, Wang M-g. Investigation of an image processing method of step-index multimode fiber specklegram and its application on lateral displacement sensing. Opt Fiber Technol 2018; 46: 48-53. DOI: 10.1016/j.yofte.2018.09.007.

[25] Fujiwara E, Marques dos Santos MF, Suzuki CK. Optical fiber specklegram sensor analysis by speckle pattern division. Appl Opt 2017; 56(6): 1585-1590. DOI: 10.1364/AO.56.001585.

[26] Crammond G, Boyd SW, Dulieu-Barton JM. Speckle pattern quality assessment for digital image correlation. Opt Lasers Eng 2013; 51(12): 1368-1378. DOI: 10.1016/j.optlaseng.2013.03.014.

[27] Aristizabal VH, Vélez FJ, Torres P. Analysis of photonic crystal fibers: Scalar solution and polarization correction. Opt Express 2006; 14(24): 11848-11854. DOI: 10.1364/OE.14.011848.

[28] Arístizabal VH, Vélez FJ, Rueda E, Gómez ND, Gómez JA. Numerical modeling of fiber specklegram sensors by using finite element method (FEM). Opt Express 2016; 24(24): 27225-27238. DOI: 10.1364/OE.24.027225.

[29] Arístizabal VH, Velez FJ, Quijano JC, Gómez JA. Numerical analysis of Fiber Specklegram stress sensors. Latin America Optics and Photonics Conference 2016: LW2C.7. DOI: 10.1364/LAOP.2016.LW2C.7.

[30] Wray JH, Neu JT. Refractive index of several glasses as a function of wavelength and temperature. J Opt Soc Am 1969; 59(6): 774-776. DOI: 10.1364/JOSA.59.000774.

[31] Aristizabal VH, Velez FJ, Torres P. Numerical model and analysis of optical fibers with internal electrodes. Rev Colomb Física 2006; 38(1): 173-176.

[32] Torres P, Aristizábal VH, Andrés MV. Modeling of photonic crystal fibers from the scalar wave equation with a purely transverse linearly polarized vector potential. J Opt Soc Am B Opt Phys 2011; 28(4): 787-791. DOI: 10.1364/JOSAB.28.000787.

\section{Authors' information}

Juan David Arango-Moreno obtained his mechatronic engineering degree in 2020, in which he served as a young researcher at the Instituto Tecnológico Metropolitano (ITM) at the Control Systems and Robotics Laboratory (LSCR), 
currently he is doing a master's degree in Automation and Industrial Control at the ITM. His research interests include computer vision, machine learning algorithms, mobile robotics, mechatronic systems, fiber optic sensors.

E-mail: juanarango220485@correo.itm.edu.co.

Yeraldin Alejandra Vélez-Galvis obtained her technology in Telecommunications at the Metropolitan Technological Institute. Currently, she is a telecommunications engineering student in the ninth semester and a young researcher at the same university. Research interests: modeling of optic sensors, design of optic systems for communication and sensing and implementation of machine learning in optic and communication systems.

Email: yeraldinvelez236975@,correo.itm.edu.co.

Victor Hugo Aristizabal-Tique obtained his B.S. degree in Physics Engineering from National University of Colombia in Medellin in 2004 and the M.Sc. in Physics in 2008 from the same university. He is currently doctoral student at Eafit University and full-time Research-Professor at the Universidad Cooperativa de Colombia, Medellín, Colombia. Research interests: analytical and computational modeling of the elastic waves in seismic engineering and geophysical prospecting, and electromagnetic waves in optical communications and sensing.

E-mails: vharisti@yahoo.com, victor.aristizabalt@.campusucc.edu.co.

Francisco Javier Vélez-Hoyos received his B.S. degree in Physics Engineering from National University of Colombia in Medellin in 2004 and the M.Sc. in Physics in 2008 from the same university. He is currently doctoral candidate at Eafit University and full-time Research-Professor at the Universidad Cooperativa de Colombia, Medellín, Colombia. Research interests: computational modeling in applied optics, sensors and elastic waves on nonclassical media.

E-mails:fjvelezh@gmail.com,francisco.velezh@,campusucc.edu.co.

Jorge Alberto Gómez-López, received his B.Sc. degree in Electrical Engineering (2002) and his M.Sc. degree in Physics (2006) from the Universidad Nacional de Colombia, Sede Medellín. He obtained his Ph.D. degree in Optical Engineering from Universidad Pontificia Bolivariana in 2012. Since 2006 he works as a full-time research Professor at Politécnico Colombiano JIC (Colombia). His current research interests involve fiber specklegram sensors and optical vortices. E-mail: jgomezlopez78@gmail.com.

Jairo Camilo Quijano-Pérez, received his B.Sc. degree in Physics Engineering (2004) and his M.Sc. degree in Physics (2007) both from the Universidad Nacional de Colombia. He obtained his Ph.D. degree in Sciences-Physics from the École Polythecnique Fédérale de Lausanne (Switzerland, 2013); he works as a full-time research Professor at Politécnico Colombiano JIC (Colombia). His current research interests involve light interaction with biological tissues and light applications for the agroindustry and sensing. E-mail: jcquijano@elpoli.edu.co.

Jorge Alexis Herrera-Ramirez (b. 1980) received his B.Sc. degree in Physics Engineering (2004) and his M.Sc. degree in Physics (2008) from the Universidad Nacional de Colombia, sede Medellín. He obtained his Ph.D. degree in Optical Engineering from Universitat Politecnica de Catalunya (Spain, 2014). During 2014, he was a Postdoctoral Fellow with the Centre for Sensors, Instruments and Systems Development of Universitat Politecnica de Catalunya. Since 2015, he became full-time Research-Professor at the Instituto Tecnologico Metropolitano de Medellin, Colombia. His current research interests involve digital holography and speckle, light modulation applications and spectral imaging. E-mail:jorgeherrerar@itm.edu.co.

Code of State Categories Scientific and Technical Information (in Russian - GRNTI)): 29.31.15, 29.33.43, 20.53.23. Received December 22, 2020. The final version-April 26, 2021. 